\title{
Hepatoid adenocarcinoma of the stomach: A case report and literature review
}

\author{
XIAO-MEI LIU ${ }^{1}$, GUI-QIU CHEN ${ }^{2}$, SHUN-LI LI ${ }^{3}$ and TIAN-SHU ZAI ${ }^{4}$ \\ ${ }^{1}$ Department of Pathology, No. 461 Clinical Department of No. 208 Hospital of PLA, Changchun, Jilin 130021; \\ ${ }^{2}$ Department of Pathology, China-Japan Union Hospital, Jilin University, Changchun, Jilin 130033; \\ ${ }^{3}$ Department of Surgery, No. 461 Clinical Department of No. 208 Hospital of PLA, Changchun, Jilin 130062; \\ ${ }^{4}$ Norman Bethune College of Medicine, Jilin University, Changchun, Jilin 130033, P.R. China
}

Received May 19, 2014; Accepted November 18, 2014

DOI: $10.3892 /$ etm.2015.2393

\begin{abstract}
Hepatoid adenocarcinoma (HAC) is a rare form of gastric cancer that has unique clinicopathological features and an extremely poor prognosis. The present case report describes a patient who was referred to the No. 461 Hospital of PLA (Changchun, China) due to nausea and occasional vomiting of acid/water. The patient was diagnosed with gastric signet-ring cell carcinoma and surgery was recommended. A total gastrectomy was performed on the operable lesion. Histopathological analysis revealed that the tumors exhibited two pathological changes: HAC and signet-ring cell carcinoma differentiation. Furthermore, the tumor cells were immunohistochemically positive for $\alpha$-fetoprotein, cytokeratin low/high molecular weight, Cam5.2, cytokeratin 7, p53, epithelial membrane antigen and carcinoembryonic antigen. Previous studies and our experience have suggested that HAC is an aggressive type of adenocarcinoma; however, radical surgery and chemotherapy may positively impact clinical outcomes.
\end{abstract}

\section{Introduction}

Hepatoid adenocarcinoma (HAC) is a type of gastric cancer with adenoid and hepatocyte differentiation. In 1985, Ishikura et al (1) first presented and stated that HAC was a cancer with hepatocyte characteristics: hepatoid adenocarcinoma of the stomach (HAS). The levels of extrahepatic $\alpha$-fetoprotein (AFP) in the serum and tumor tissue are high in this form of gastric cancer, similarly to hepatocellular

Correspondence to: Professor Gui-Qui Chen, Department of Pathology, China-Japan Union Hospital, Jilin University, 126 Xiantai Street, Changchun, Jilin 130033, P.R. China

E-mail: cd781214@gmail.com

Professor Xiao-Mei Liu, Department of Pathology, No. 461 Clinical Department of No. 208 Hospital of PLA, 108 Ziyou Road, Changchun, Jilin 130021, P.R. China

E-mail: liuxiaomei@gmail.com

Key words: hepatoid adenocarcinoma, stomach carcinoma (HCC). Since the study by Ishikura et al, there have been subsequent studies published on the clinical manifestation and pathological features of HAC (2-4). This tumor type has poor prognosis and therefore accurate diagnosis of HAC is extremely important. The first description of HAC was in the stomach, which remains the most common tumor location; however, HAC has been reported to develop in a variety of organs including the gallbladder, lungs, bladder, esophagus, pancreas, peritoneum, jejunum, colon, rectum, renal pelvis, ureter, ovaries, uterus and papilla of Vater (5-8). HAS is a relatively rare gastric carcinoma and has an extremely poor diagnosis. Only a few cases have been previously reported. The present case report describes a patient who experienced acid regurgitation, belching, nausea and occasional vomiting of acid/water, which was more notable on an empty stomach. No diarrhea or abdominal distension was observed but the patient's stools were black.

\section{Case report}

A 47-year-old male presented with repetitive upper abdominal ache, without obvious inducement, that had lasted three and a half months. The ache was accompanied with acid regurgitation and belching, which was more notable on an empty stomach. The patient had nausea and occasional acid vomiting but no diarrhea or abdominal distension. The patient's stools were black. Following gastroscopy and pathological examination, the patient was diagnosed with gastric signet-ring cell carcinoma and a total gastrectomy was performed on the operable lesion. Abdominal ultrasonography showed normal results in the liver, gallbladder, pancreas, spleen and kidney, and CEA levels were normal. The patient did not have lymph node metastasis or other organ metastasis. Thus, 2 weeks following surgery the patient left hospital without undergoing chemotherapy or radical therapy. $\mathrm{He}$ is currently being followed up for 12 months. Informed consent was obtained from the patient and the patient's family prior to participation in the present study.

\section{Pathological examination}

Naked eye. The whole stomach was inspected: The greater gastric curvature measured $26 \mathrm{~cm}$ and the lesser gastric curvature measured $14 \mathrm{~cm}$. A $2.3 \times 2.2 \times 2.0-\mathrm{cm}$ nodule was 


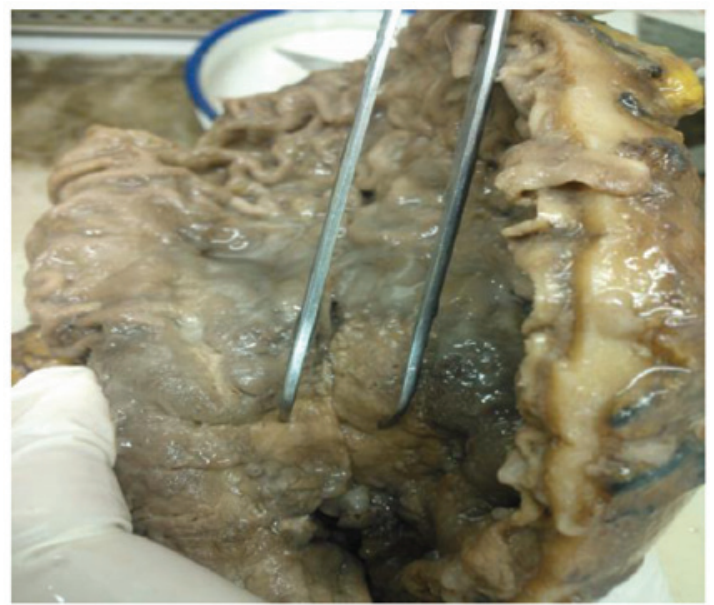

Figure 1. Neoplasm on the lesser gastric curvature side.

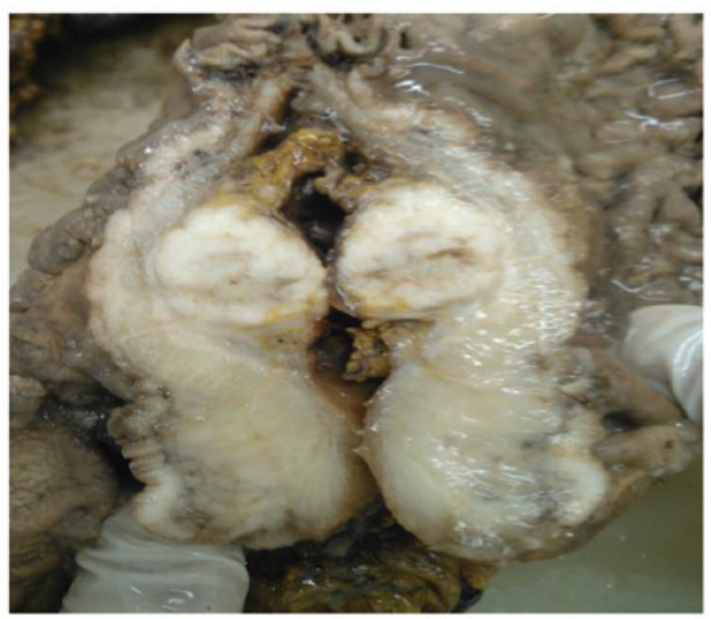

Figure 2. Neoplasm on the subserosal surface.

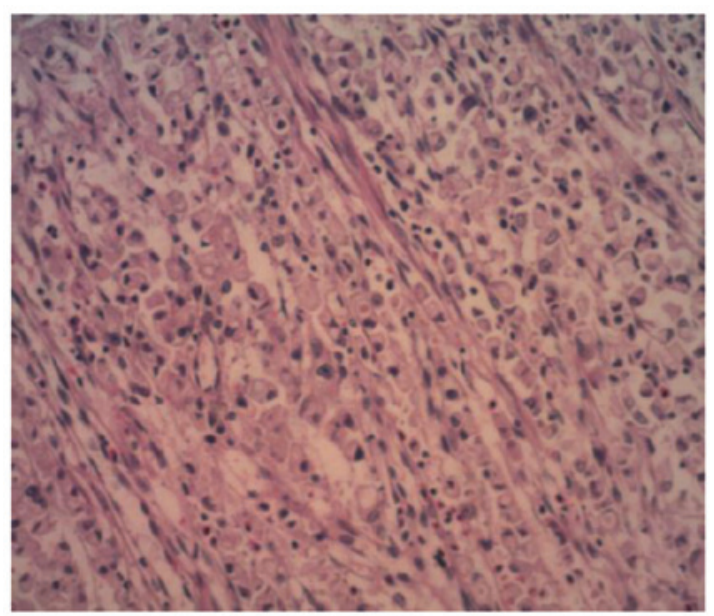

Figure 3. Signet-ring cell carcinoma (hematoxylin and eosin staining; magnification, $\mathrm{x} 100)$

identified on the serosal surface. On incision along the greater curvature side, a $4.5 \times 3.5 \times 0.4 \mathrm{~cm}$ ulcerative neoplasm was found $4.5 \mathrm{~cm}$ from the pylorus and $6.0 \mathrm{~cm}$ from the gastric cardia, on the lesser gastric curvature side (Fig. 1). Following

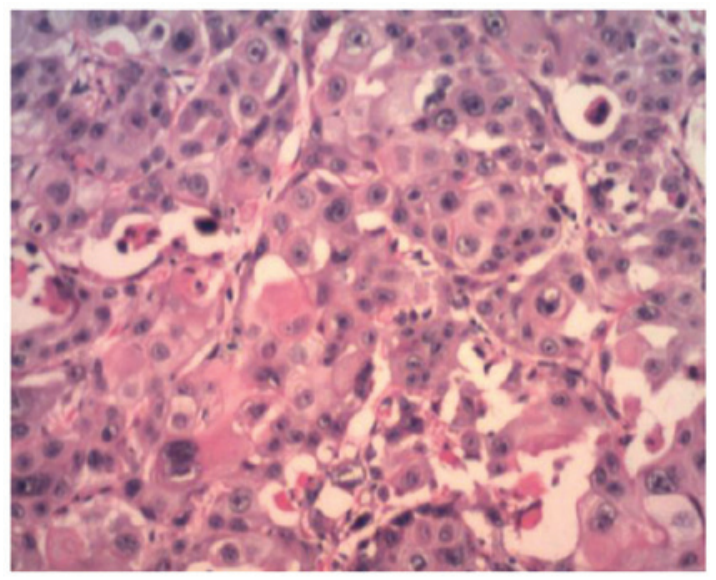

Figure 4. Hepatic adenocarcinoma (hematoxylin and eosin staining; magnification, $\mathrm{x} 400$ ).

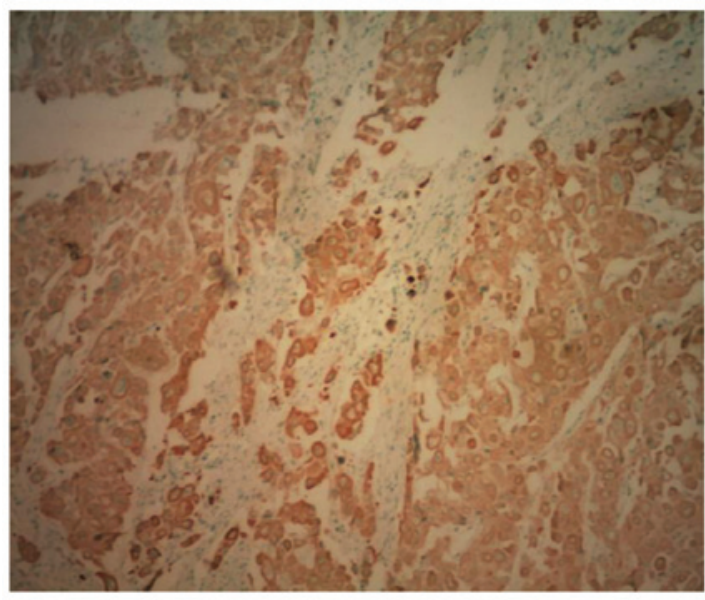

Figure 5. Immunohistochemical staining for $\alpha$-fetoprotein (strongly positive) in the heptatoid adenocarcinoma. Magnification, x100.

incision along the ulcerative neoplasm, it was observed that the section was brown and gray, hard and had invaded the shallow muscularis. An isolated circular nodule, which was $2.0 \mathrm{~cm}$ in diameter, gray and hard, with clear boundaries (Fig. 2).

Microscopic evaluation. The mucosa of the neoplasm showed features consistent with signet-ring cell carcinoma (Fig. 3); however, large cells arranged in a solid, clumped shape were observed on the serosa of the neoplasm. The nuclei were large and centrally located. Nucleolus and nuclear fission were observed. The cytoplasm was abundant, acidophilic and transparent. A small amount of fibrous tissue, capillaries and a Periodic-Acid Schiff (PAS)-positive corpuscle with necrosis were also observed (Fig. 4).

Immunohistochemistry (IHC). Positive results for cytokeratin low/high molecular weight (AE1/AE3), Cam5.2, cytokeratin 7, p53, epithelial membrane antigen (EMA) and carcinoembryonic antigen (CEA) were found on both sides of the neoplasm. Staining for AFP was strongly positive in the neoplasm at the serosa (Fig. 5). Tests for neuroendocrine markers (synaptophysin, chromogranin A and cluster of differentiation 56), mesothelioma markers (calretinin, D240 and mesothelial cells), markers of the gastric choriocarcinoma 
( $\beta$-human chorionic gonadotrophin and inhibin) and a marker of squamous cell carcinoma (p63) were negative.

Pathological diagnosis. The patient was diagnosed with gastric adenocarcinoma combined with HAC and signet-ring cell carcinoma differentiation. The signet-ring cell carcinoma invaded the middle muscularis. Three out of the 19 lymph nodes on the greater gastric curvature were identified as metastases and one of the 10 lymph nodes on the lesser gastric curvature was a metastasis. All metastases were signet-ring cell carcinoma.

\section{Discussion}

HAC is a type of gastric cancer with adenoid and hepatocyte differentiation. In 1985, Ishikura et al (1) first presented and stated unequivocally that HAC was a cancer with hepatocyte characteristics. Levels of AFP in the serum and tumor tissue are significantly high in this form of gastric cancer; however, $\mathrm{HAC}$ is rare, accounting for $1.3-15 \%$ of gastric cancers (9). Although there have been some previous reports about this disease $(10,11)$, there remains a lack of understanding. HAC is discussed here with reference to the present case and published literature.

HAC, an aggressive and highly malignant tumor, is a type of adenocarcinoma with the structural and cytological characteristics of HCC that occurs in extrahepatic organs or tissues. In cases of HAC, high levels of AFP can be detected in the serum and tumor tissue. HAC usually occurs in the stomach; however, the tumors may also be observed in the duodenum, colon, rectum, esophagus, gallbladder, pancreas, lung, bladder, kidney, ureter, ovaries, uterus, retroperitoneum and local soft tissue (12). HAS occurs more frequently than the other types of HAC, generally occurs in middle-aged patients and is more commonly found in males than females. It is likely that it originates in the gastric antrum and is associated with infiltration and ulceration. The tumor locates itself in the submucosa and infiltrates to the muscularis layer. HAS generally has no specific clinical symptoms, and the digestive tract symptoms, such as abdominal pain, abdominal distension and black stools, are often apparent in the middle and late periods of the disease. HAC is frequently associated with an elevated serum AFP and positive staining for AFP in IHC tests. Postoperative serum AFP levels may be used as a predictive index for tumor recurrence or metastasis (13). HAC is additionally prone to spread to the liver and lymph nodes, since it is similar to liver cancer cells in histology. A thrombus is easily formed and can invade the vascular system early on due to a rich blood supply. In certain studies, the blood and lymphatic vessel invasion by HAS and the level of surrounding lymph node metastasis have been observed to be evidently higher than those associated with a less-differentiated gastric adenocarcinoma $(14,15)$; however, in the present case, the lymph node metastasis was signet-ring cell carcinoma rather than HAC, which was not in conformity with the literature.

Pathologically, HAC comprises primary tumors in the gastric mucosa gland. The tumor cells generally form two different but closely associated areas: Adenocarcinoma and hepatocellular cancer. The former is a poorly differentiated, visible tubular and papillary adenocarcinoma $(5,16)$. The latter exhibits HCC differentiation. The pathology and IHC of HAC show numerous features. Under a microscope, the cancer cells are large with abundant cytoplasm, and the nuclei are round or ovoid. The nucleolus and mitotic spindle can be observed and the cytoplasm exhibits pale eosin staining (17). A small number of cancer cells (transparent and acidophilic polygonal cells) form the medullary or cable structure. These cells are separated by a few fibrous tissues and a rich blood supply, and exhibit transitional or overlapping processes. Visible eosinophilic hyaline PAS-positive corpuscles lie between the cells. The cancer cells may show different degrees of liver cell differentiation, characterized by fatty degeneration or the secretion of bile. Under the electron microscope, a capillary bile duct structure may be observed between tumor cells. The two areas of HAC both exhibit intestinal epithelial microvilli, which come from the gastrointestinal epithelium. In IHC, tests for AFP are strongly positive or positive, tests for CEA may be positive or negative and tests for $\alpha 1$-antitrypsin and $\alpha 1$-antichymotrypsin are positive in the liver cancer region. AFP expression is strongly positive on the serosal surface. According to the results of AFP IHC staining, HAC can be divided into AFP-positive and AFP-negative types (18); however based on the characteristics of structural organization, HAC can be divided into simple (only the structure of liver cancer) and mixed (adenocarcinoma and HCC) types. In the current case, the HAC was an AFP-positive, mixed type.

It is important to monitor the increase in serum AFP level and the expression of AFP in tumor tissues when studying HAC. Patients with HAC have higher serum AFP levels. It is generally believed that this may be due the following factors: i) The liver-differentiated area of the HAC produces AFP; ii) the gastric cancer itself produces AFP instead of the liver-differentiated area; iii) the gastric cancer metastasizes to the liver and AFP is then produced by the new or proliferating hepatocytes (19). Inagawa et al (2) reported that the serum AFP level was associated with the differentiation degree, and Chang et al (20) found that a higher serum AFP level resulted in a poorer prognosis than that for AFP-negative gastric cancer. Nagai et al (21) suggested that patients with HAC with a high or normal AFP had the same prognosis.

Gastric cancer is complex and highly heterogeneous. There have been few studies regarding the differences between the signet-ring cell carcinoma and HAC. Akiyama et al (22) found that tubular adenocarcinoma and HAC had the same origin following the technical application of molecular pathology. Both carcinomas had X chromosome and p53 loss; therefore we hypothesize that they have the same origin. Tumors from the same origin can have different biological behavior, morphology and IHC profiles, which fully reflect the heterogeneous characteristics of tumor growth. This feature makes the treatment of such tumors difficult, due to their particularly complex biological behavior and poor prognosis.

In summary, the present study described an unusual case of HAC which had a morphological similarity to HCC. On initial observation, the elevation in the level of AFP was caused by HCC; however, the second spike in the level of AFP was due to HAC. Thus, a differential diagnosis of HAC should be considered in cases involving the elevation of the levels of serum AFP, even in patients at a high risk of HCC. 


\section{References}

1. Ishikura H, Fukasawa Y, Ogasawara K et al: An AFP-producing gastric carcinoma with features of hepatic differentiation. A case report. Cancer 56: 840-848, 1985.

2. Inagawa S, Shimazaki J, Hori M et al: Hepatoid adenocarcinoma of the stomach. Gastric Cancer 4: 43-52, 2001.

3. Liu X, Cheng Y, Sheng W, et al: Analysis of clinicopathologic features and prognostic factors in hepatoid adenocarcinoma of the stomach. Am J Surg Pathol 34: 1465-1471, 2010.

4. Ye MF, Tao F, Liu F and Sun AJ: Hepatoid adenocarcinoma of the stomach: a report of three cases. World J Gastroenterol 19: 4437-4442, 2013.

5. Su JS, Chen YT, Wang RC, et al: Clinicopathological characteristics in the differential diagnosis of hepatoid adenocarcinoma: a literature review. World J Gastroenterol 19: 321-327, 2013.

6. Yano T, Ishikura H, Wada T, et al: Hepatoid adenocarcinoma of the pancreas. Histopathology 35: 90-92, 1999.

7. Ishikura H, Ishiguro T, Enatsu C, et al: Hepatoid adenocarcinoma of the renal pelvis producing alpha-fetoprotein of hepatic type and bile pigment. Cancer 67: 3051-3056, 1991.

8. Gardiner GW, Lajoie G and Keith R: Hepatoid adenocarcinoma of the papilla of Vater. Histopathology 20: 541-544, 1992.

9. Chang YC, Nagasue N, Kohno H et al: Clincicopathologic features and long-term results of alpha-fetoprotein-producing gastric cancer. Am J Gastroenterol 85: 1480-1485, 1990.

10. Baek SK, Han SW, Oh DY, et al: Clinicopathologic characteristics and treatment outcomes of hepatoid adenocarcinoma of thestomach, a rare but unique subtype of gastric cancer. BMC Gastroenterol 11: 56, 2011.

11. Giuffrè G, Ieni A, Barresi V, Caruso RA and Tuccari G: HER2 status in unusual histological variants of gastric adenocarcinomas. J Clin Pathol 65: 237-241, 2012.

12. Rotellini M, Messerini L, Stomaci N and Raspollini MR: Hepatoid adenocarcinoma of the ureter: unusual case presenting hepatic and ovarian metastases. Appl Immunohistochem Mol Morphol 19: 478-483, 2011
13. deLorimier A, Park F, Aranha GV and Reyes C: Hepatoid carcinoma of the stomach. Cancer 71: 293-296, 1993.

14. Ishikura $\mathrm{H}$, Kishimoto $\mathrm{T}$, Andachi $\mathrm{H}$ et al: Gastrointestinal hepatoid adenocarcinoma: venous permeation and mimicry of hepatocellular carcinoma, a report of four cases. Histopathology 31 : 47-54, 1997.

15. Kang GH and Kim YI: Alpha-fetoprotein-producing gastric carcinoma presenting focal hepatoid differentiation in metastatic lymph nodes. Virchows Arch 432: 85-87, 1998.

16. Jalle T, Gérard C, Lada PE, et al: Hepatoid adenocarcinoma of the stomach. A case report. Ann Chir 131: 213-215, 2006 (In French).

17. Cappetta A, Bergamo F, Mescoli C, Lonardi S, Rugge M and Zagonel V: Hepatoid adenocarcinoma of the colon: what should we target? Pathol Oncol Res 18: 93-96, 2012.

18. Ishikura $\mathrm{H}$, Kirimoto $\mathrm{K}$, Shamoto $\mathrm{M}$ et al: Hepatoid adenocarcinoma of the stomach. An analysis of seven cases. Cancer 58: 119-126, 1986

19. Rassidakis GZ, Delladetsima JK, Letsos SP et al: Hepatoid adenocarcinoma of the stomach with extensive neuroendocrine differentiation and a coexisting carcinoid tumour. Histopathology 33: 186-188, 1998.

20. Chang YC, Nagasue $\mathrm{N}$, Abe $\mathrm{S}$ et al: Comparison between the clinicopathologic features of AFP-positive and AFP-negative gastric cancers. Am J Gastroenterol 87: 321-325, 1992.

21. Nagai E, Ueyama $T$, Yao $T$ and Tsuneyoshi M: Hepatoid adenocarcinoma of the stomach: A clinicopathologic and immunohistochemical analysis. Cancer 72: 1827-1835, 1993.

22. Akiyama S, Tamura G, Endoh Y et al: Histogenesis of hepatoid adenocarcinoma of the stomach: molecular evidence of identical origin with coexistent tubular adenocarcinoma. Int J Cancer 106: $510-515,2003$. 